Review

\title{
Thermotropic and Barotropic Phase Behavior of Phosphatidylcholine Bilayers
}

\author{
Hitoshi Matsuki $^{1, *}$, Masaki Goto ${ }^{1}$, Kaori Tada $^{2}$ and Nobutake Tamai ${ }^{1}$
}

1 Department of Life System, Institute of Technology and Science, The University of Tokushima, 2-1 Minamijosanjima-cho, Tokushima 770-8506, Japan;

E-Mails: goto@bio.tokushima-u.ac.jp (M.G.); tamai@bio.tokushima-u.ac.jp (N.T.)

2 Department of Material Science and Technology, Kochi National College of Technology, 200-1 Monobe-otsu, Nankoku, Kochi 783-8508, Japan; E-Mail: tada@ge.kochi-ct.ac.jp

* Author to whom correspondence should be addressed; E-Mail: matsuki@bio.tokushima-u.ac.jp; Tel.: +81-88-656-7513; Fax: +81-88-655-3162.

Received: 21 December 2012; in revised form: 11 January 2013 / Accepted: 15 January 2013 / Published: 24 January 2013

\begin{abstract}
Bilayers formed by phospholipids are frequently used as model biological membranes in various life science studies. A characteristic feature of phospholipid bilayers is to undergo a structural change called a phase transition in response to environmental changes of their surroundings. In this review, we focus our attention on phase transitions of some major phospholipids contained in biological membranes, phosphatidylcholines (PCs), depending on temperature and pressure. Bilayers of dipalmitoylphosphatidylcholine (DPPC), which is the most representative lipid in model membrane studies, will first be explained. Then, the bilayer phase behavior of various kinds of PCs with different molecular structures is revealed from the temperature-pressure phase diagrams, and the difference in phase stability among these PC bilayers is discussed in connection with the molecular structure of the PC molecules. Furthermore, the solvent effect on the phase behavior is also described briefly.
\end{abstract}

Keywords: bilayer; lipid membrane; phase diagram; phase transition; phosphatidylcholine; pressure 


\section{Introduction}

Biological membranes consist mainly of phospholipid and protein molecules arranged in organized but flexible sheets. The phospholipid components form a closed bilayer aggregate, which provides a basic structure of biological membranes, because they are amphiphilic molecules. The most noteworthy property of phospholipid bilayers is to undergo the structural change of the bilayer, called a phase transition, in response to environmental changes of their surroundings such as temperature, pressure, salts, $\mathrm{pH}$ and solvents. The melting phenomenon of fatty-acid chains, that is, trans-gauche conformational change of phospholipid molecules in the bilayers with increasing temperature, is a representative phase transition. The transition has been analyzed a great deal by lipid researchers by means of various kinds of physico-chemical methods [1,2]: macroscopic approaches, such as differential scanning calorimetry (DSC); microscopic approaches, such as X-ray and neutron diffraction; and, approaches by statistical mechanics, such as molecular simulation [3].

Although a number of studies on the phase transitions of phospholipid bilayers have been reported [4], most of them are performed as a function of temperature and the concentration or additive concentrations under atmospheric pressure. There are only a few studies regarding pressure effect on the bilayers, and information on the phase behavior under high pressure is also lacking. Because phospholipid bilayers respond non-isotropically to changes in the environmental variables (temperature, pressure, etc.), it is interesting to examine the pressure effect on the bilayer. For example, in a gel state, the phospholipid molecule extends in the direction of bilayer normal (increase in membrane thickness) and shrinks in the direction of bilayer parallel (decrease in cross-sectional area), whereas the situation is reversed in the liquid crystalline state [5]. Therefore, the application of uniform and isotropic hydrostatic pressure on the bilayers brings about great mechanical fluctuation in them, and then the membranes change the phase state to take the most stable one under the pressure, which leads to a non-observable state under atmospheric pressure.

In many phospholipid species, the bilayer properties of phosphatidylcholines (PCs), which are major constituents in biological membranes of eukaryotic cells, have already been widely investigated [6,7]. The molecular structure of PC is shown in Figure 1a. On the other hand, studies on phospholipid bilayers under high pressure are considerably limited. Most of the pressure studies have concentrated on the bilayer properties of a certain phospholipid, dipalmitoylphosphatidylcholine (DPPC) [8-15], which is a symmetric PC with two equivalent palmitoyl chains as shown in Figure $1 \mathrm{~b}$. This seems to originate from the fact that DPPC has the maximum percentage in symmetric PCs (diacyl-PCs) in biological membranes such as erythrocyte membranes and that the bilayer is markedly desirable as model biological membranes because a gel-to-liquid-crystalline transition in the bilayer and an expanded-condensed transition in the insoluble monolayer can be easily observed at ambient temperatures experimentally. We have started the pressure study on phospholipid bilayers with the DPPC bilayer and investigated the phase behavior of various kinds of PC bilayers. In this review, we explain the bilayer properties of DPPC under atmospheric and high pressure in the first place, and then we show the thermotropic and barotropic phase behavior of bilayers of various kinds of PCs with different molecular structures. Furthermore, we also describe the solvent effect on the phase behavior of the DPPC bilayer. 
Figure 1. (a) Molecular structure of PC. (b) Three-dimensional picture of DPPC.

(a)

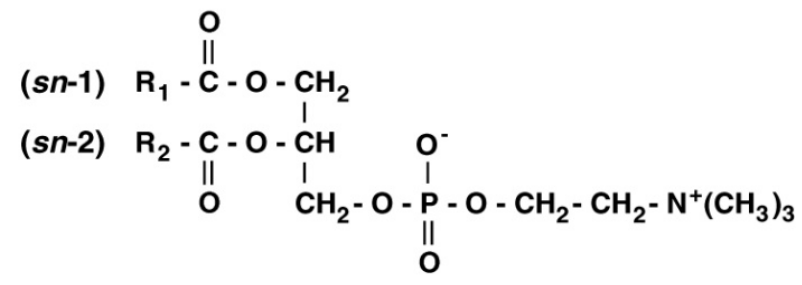

(b)

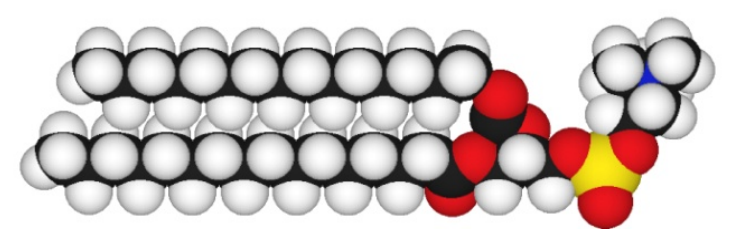

\section{Lyotropic, Thermotropic and Barotropic Phase Transitions of DPPC Bilayer}

A solid crystal of a phospholipid has a well-ordered bilayer aggregate with lamellar structures [16]. When adding water to the solid crystal, water molecules hydrate the polar head group of the lipid molecule. The added water molecules are accumulated in the interlayer between bilayers and the lamellar structures are swollen. Since there is a limit of the thickness of the interlayer between bilayers (e.g., ca. 10 molecules for PC in the gel phase [17]), excess water molecules separate from the lamellar structures and become bulk water molecules. A bilayer aggregate formed by the hydration is a closed microsome with a diameter from about $20 \mathrm{~nm}$ to about $1000 \mathrm{~nm}$, although the diameter is dependent on the molecular structure of the phospholipid and the preparation method. This closed bilayer aggregate with unilamellar or multilamellar structure formed by phospholipid molecules in aqueous solutions is referred to as a vesicle or a liposome.

The bilayers of phospholipids undergo lyotropic phase transitions by hydration of water molecules. The lyotropic phase transitions of PC bilayers have been investigated in detail as a function of temperature $[18,19]$. The temperature-water content phase diagram of the DPPC bilayer is shown in Figure $2 \mathrm{a}$ together with the membrane state of each phase. The membrane states induced by hydration are: hydrated crystal or subgel $\left(\mathrm{L}_{\mathrm{c}}\right)$ phase, gel $\left(\mathrm{L}_{\beta}\right)$ phase, and liquid crystalline $\left(\mathrm{L}_{\alpha}\right)$ phase. The melting point of nonhydrated crystal of DPPC $\left(c a .115^{\circ} \mathrm{C}\right)$ drastically decreases with increasing water content, up to $c a$. $10 \%$ of water content. However, exceeding $10 \%$, a phase transition originating from hydration change in polar head groups appears at a low temperature in addition to the chain melting transition at a high temperature. Furthermore, at $15 \%$ of water content, another phase appears in the gel phase at temperatures between the above two phase-transition temperatures due to the fluctuation of molecular packing in the bilayer. These phase transitions are called the sub-, pre- and main transitions from the low temperature and correspond to the transition between the $\mathrm{L}_{\mathrm{c}}$ phase and the gel (lamellar gel $\left.\left(\mathrm{L}_{\beta}^{\prime}\right)\right)$ phase, the transition between the gel $\left(\mathrm{L}_{\beta}^{\prime}\right.$ and ripple gel $\left.\left(\mathrm{P}_{\beta}^{\prime}\right)\right)$ phases, the transition between the gel $\left(\mathrm{P}_{\beta}^{\prime}\right)$ phase and the $\mathrm{L}_{\alpha}$ phase, respectively. Here, the superscript of prime means that lipid molecules are oriented on the tilt from the bilayer plane. The tilted orientation of lipid molecules in bilayers is one of characteristics for the bilayers of PCs with saturated acyl chains. Three kinds of phase-transition temperatures become almost constant, irrespective of water content beyond ca. $25 \%$. Therefore, although lipid concentrations are considerably different in individual lipid-membrane 
studies, depending on the measurement methods such as DSC ( $>99 \%$ of water content) and X-ray diffraction $(60 \%-95 \%$ of water content), the observed phase states in these methods are the same as each other.

In the region where the phase-transition temperatures are not affected by water content, we can specify the phase states of phospholipid bilayers. By adopting pressure $(p)$ as an experimental variable together with temperature $(T)$, we have investigated not only the thermotropic but also the barotropic phase transitions of various PC bilayers using DSC under atmospheric pressure and optical measurement methods under high pressure and constructed the $T-p$ phase diagrams by using the obtained phase-transition temperatures and pressures. The detailed experimental techniques for the determination of the bilayer phase transitions and the discussion about thermodynamic quantities of the phase transition are described in the references given in the following sections. Figure $2 \mathrm{~b}$ demonstrates the $T-p$ phase diagram of the DPPC bilayer [20]. The three kinds of phase-transition temperatures increase linearly by applying pressure. A notable feature of the DPPC bilayer is the formation of nonbilayer structure, the pressure-induced interdigitated gel $\left(\mathrm{L}_{\beta} \mathrm{I}\right)$ phase, observed at high pressures above $c a$. $100 \mathrm{MPa}$. In the $\mathrm{L}_{\beta} \mathrm{I}$ phase, the acyl chains of the DPPC molecule on a monolayer of one side of a bilayer interpenetrate alternatively into those on a monolayer of the other side of a bilayer. Further pressurization brings about the extension of the $\mathrm{L}_{\beta} \mathrm{I}$-phase region. The polymorphism of the gel phases such as the $\mathrm{L}_{\beta}{ }^{\prime}$ and $\mathrm{P}_{\beta}$ ' phases with tilted molecular orientation and the nonbilayer $\mathrm{L}_{\beta} \mathrm{I}$ phase is characteristic of bilayers of PCs with a bulky choline head group. This is attributable to the large steric hindrance among adjacent choline groups.

Figure 2. (a) Effect of water content on phase-transition temperatures of DPPC bilayer. The membrane state of each phase is also depicted in the figure. (b) Temperature-pressure phase diagrams of DPPC bilayer. Phase transitions: (green) subtransition, (blue) pretransition and transitions between gel phases, (red) main transition. Dashed line in figure (b) indicates the transition between metastable gel phases.
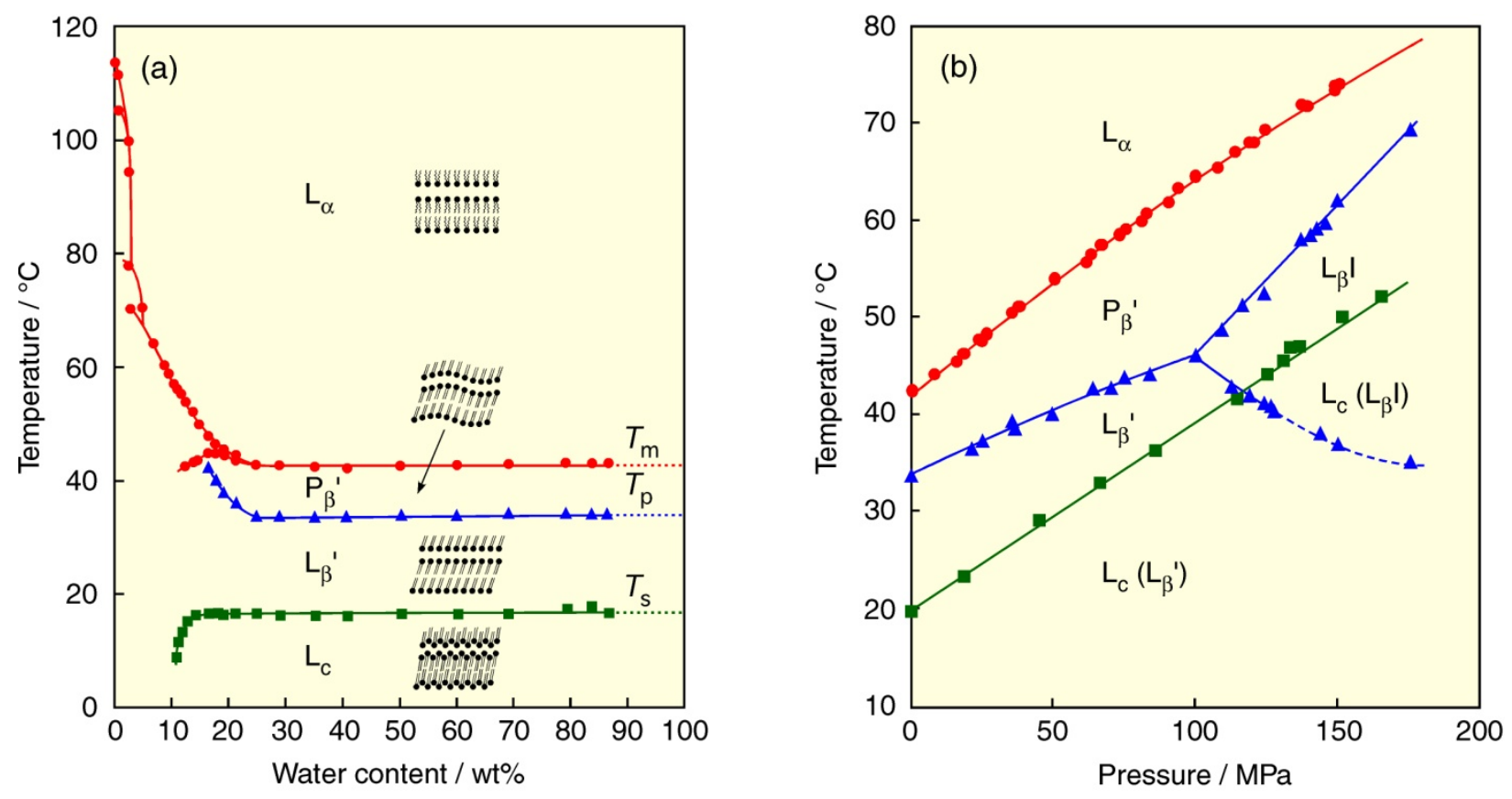


\section{Effect of Acyl Chain Structure on the Phase Behavior of PC Bilayer Membranes}

So many species of phospholipids can be obtained by combining hydrophobic fatty-acid chains with different chain length or degree of unsaturation with several kinds of polar head groups. Here, we systematically explain the $T-p$ phase diagrams of bilayers formed by various PCs that can be obtained by changing the molecular structure of DPPC.

\subsection{Length of Acyl Chain}

The $T-p$ phase diagrams for the bilayers of diacylphosphatidylcholines (CnPC: $n=14-18)$, which are symmetric PCs with saturated acyl chains of carbon number of $n$ and homologs of DPPC (C16PC), are illustrated in Figure 3a [21]. Here the subtransition lines are omitted from the figure for simplicity. The phase diagrams of all $\mathrm{Cn} \mathrm{PC}$ bilayers are similar in shape although the diagram moves to the low-pressure and high-temperature region with increasing $n$. The similarity among the diagrams indicates that the slope $(\mathrm{d} T / \mathrm{d} p)$ of each phase transition is similar to each other irrespective of the acyl-chain length. We revealed that the slopes of the phase boundaries depend on the kinds of the transitions and have similar values if the transition is the same [20-22]. With increasing acyl-chain length of the PC molecule, both the pre- and main-transition temperatures elevate [21-26]. Namely, the regions of the $L_{\alpha}$ and $P_{\beta}$ ' phase shrink (destabilize), while those of the $L_{\beta}{ }^{\prime}$ and $L_{\beta} I$ phase extend (stabilize) in the order of the acyl-chain length of the PC molecule. It is understandable that the bilayer becomes more rigid in proportion to the magnitude of van der Walls interaction in the PC molecules. Furthermore, it is noted that the pressure of the triple point corresponding to the coexisting three gel $\left(\mathrm{L}_{\beta}{ }^{\prime}, \mathrm{P}_{\beta}{ }^{\prime}\right.$, and $\left.\mathrm{L}_{\beta} \mathrm{I}\right)$ phases steeply decreases with the chain elongation: the $\mathrm{L}_{\beta} \mathrm{I}$ phase remarkably extends the region to lower pressures. We call the pressure of the triple point the minimum interdigitation pressure (MIP).

Figure 3. (a) Temperature-pressure phase diagrams of diacyl-PC ( $\mathrm{CnPC}$ ) bilayers: (1) C14PC (DMPC), (2) C15PC, (3) C16PC (DPPC), (4) C17PC, (5) C18PC (DSPC).

(b) Temperature-pressure phase diagrams of $\mathrm{CnPC}$ bilayers: (1) C19PC, (2) C20PC, (3) C21PC, (4) C22PC. Phase transitions: (blue) pretransition and transitions between gel phases, (red) main transition.
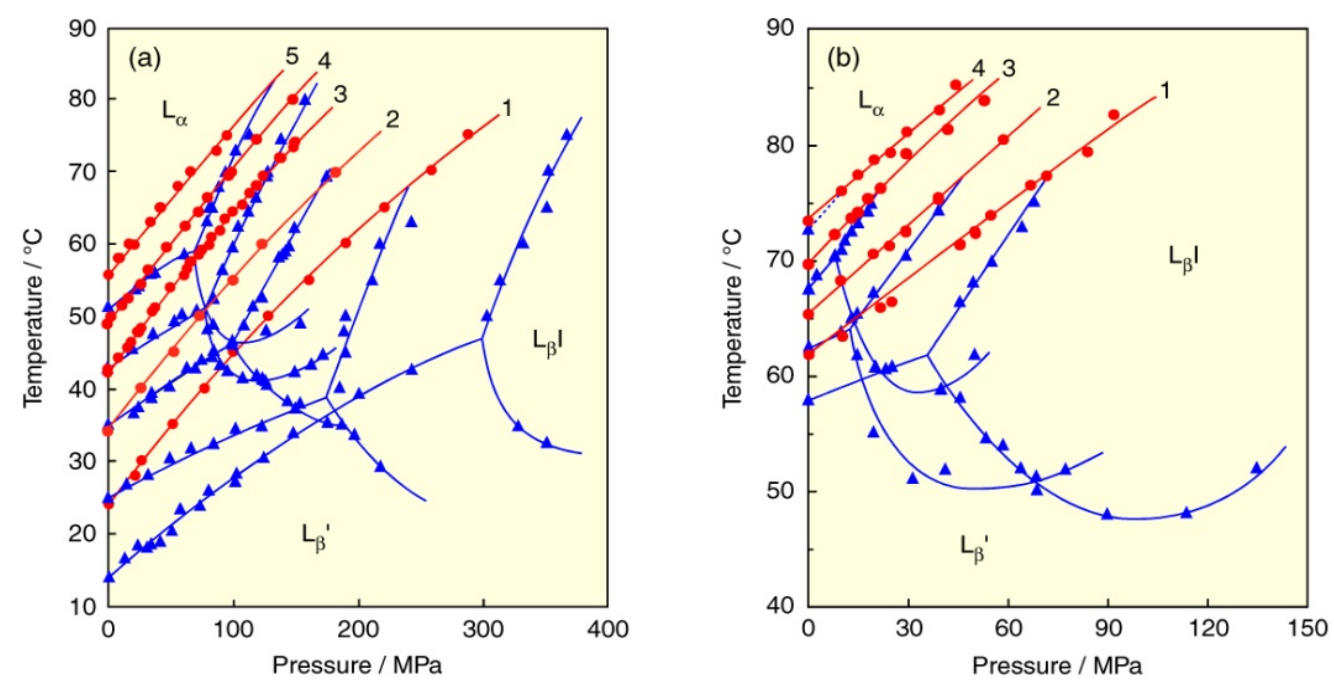
We have recently examined the phase behavior of symmetric PCs with longer saturated acyl chains with a carbon number of 19 and over. Figure $3 \mathrm{~b}$ shows the $T-p$ phase diagrams constructed for the bilayers of symmetric PCs with saturated long acyl chains (CnPC: $n=19-22)$. With increasing acyl-chain length, the $L_{\alpha}$ and $P_{\beta}^{\prime}$ phases further shrink the regions while the $L_{\beta}{ }^{\prime}$ and $L_{\beta} I$ phases further extend the regions and the MIP values decrease more and more. Interestingly, the phase boundary between the $\mathrm{L}_{\beta}$ ' and $\mathrm{L}_{\beta} \mathrm{I}$ phases disappears in the case of the $\mathrm{C} 22 \mathrm{PC}$ bilayer, and hence there is no triple point in the diagram. The phase behavior of the C22PC bilayer resembles those of ether-linked PC bilayers described in the following section. It can be said that the $\mathrm{C} 22 \mathrm{PC}$ bilayer can induce the $\mathrm{L}_{\beta} \mathrm{I}$ phase even under atmospheric pressure. We confirmed the interdigitation of the C22PC bilayer under atmospheric pressure by the neutron scattering and fluorometry under high pressure [27]. The interdigitation of the $\mathrm{C} 22 \mathrm{PC}$ bilayer by only hydration suggests that the attractive interaction between acyl chains due to the hydrophobicity is a dominant factor for the bilayer interdigitation, as well as the repulsive interaction among polar head groups.

Figure 4. (a) Temperature-pressure phase diagrams of $\mathrm{Cn} P C$ bilayers: (1) $\mathrm{C} 12 \mathrm{PC}$ (DLPC), (2) C13PC. Phase transitions: (blue) pretransition of C13PC bilayer, (red) main transition. (b) Temperature-pressure phase diagrams of C12PC bilayer in $50 \mathrm{wt} . \% \mathrm{EG}$ solution. Phase transitions: (green) subtransition, (blue) pretransition and transitions between gel phases, (red) $\mathrm{P}_{\beta}^{\prime} / \mathrm{L}_{\alpha}$ or $\mathrm{P}_{\beta}^{\prime} / \mathrm{L}_{\mathrm{x}}$ transition, (green) $\mathrm{L}_{\mathrm{c}} / \mathrm{L}_{\mathrm{x}}$ or $\mathrm{L}_{\mathrm{c}} / \mathrm{P}_{\beta}{ }^{\prime}$ transition, (black) $\mathrm{L}_{\mathrm{x}} / \mathrm{L}_{\alpha}$ transition.
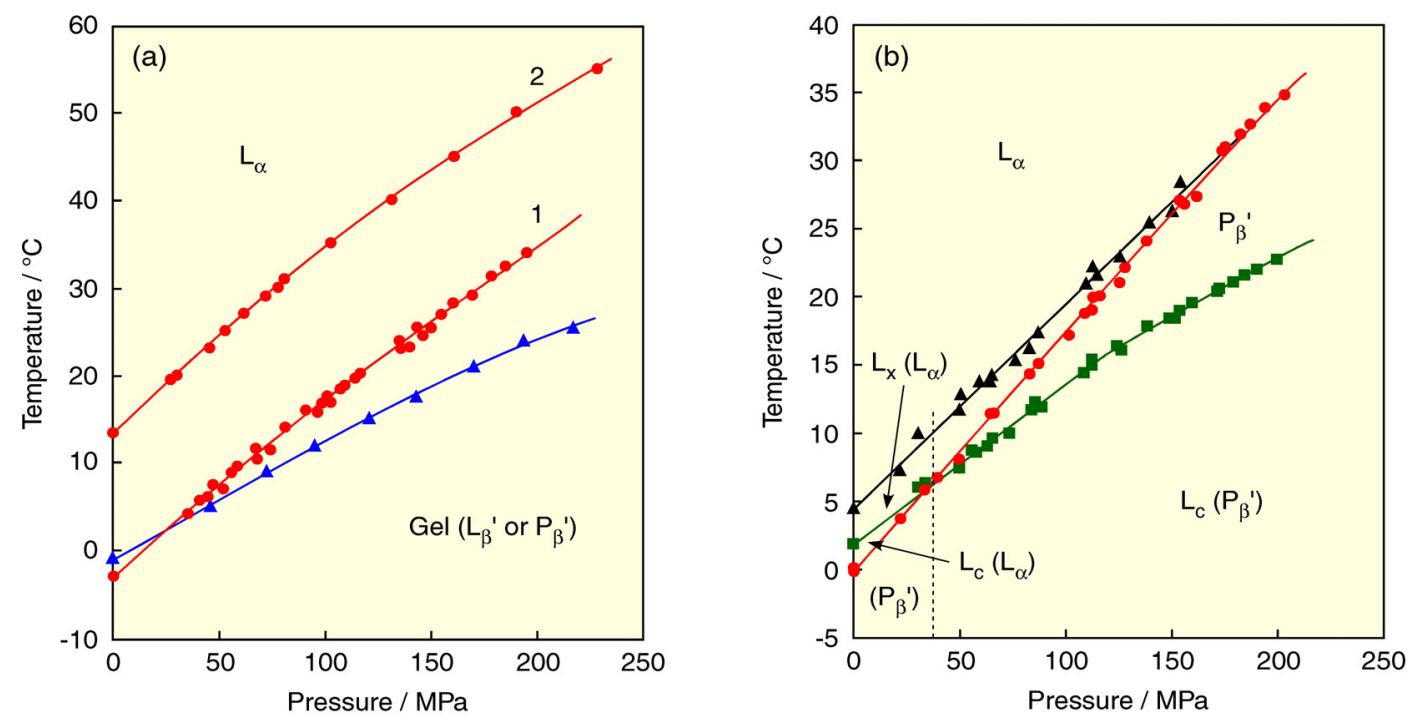

Meanwhile, we have also investigated the thermotropic and barotropic bilayer phase behavior of symmetric PCs with shorter saturated acyl chains. Our consideration on the thermodynamic quantities of the bilayer phase transitions revealed that the main transition occurs in the bilyers of PCs with acyl chains of a carbon number of 12 and over [22] and the bilayer interdigitation does in those of PCs with the chains of a carbon number of 14 and over [21]. Figure 4a depicts the $T-p$ phase diagrams of symmetric PCs with shorter chains (CnPC: $n=12,13)$ [21,22]. Unlike the bilayers of PCs with longer chains, the $\mathrm{L}_{\alpha}$ phase region is more extended due to the depression in the main-transition temperature. The pretransition is no longer observed in the C12PC bilayer. Since it is reported [28,29] that the $\mathrm{C} 12 \mathrm{PC}$ bilayer exhibits different phase behavior from other $\mathrm{Cn} \mathrm{PC}$ bilayers, we have investigated the 
behavior by using aqueous ethylene glycol (EG) solutions [30]. Here, EG was used as an antifreeze in order to avoid the water freezing, because the C12PC bilayer undergoes the main transition at a temperature below $0{ }^{\circ} \mathrm{C}$. The phase diagram of the $\mathrm{C} 12 \mathrm{PC}$ bilayer in $50 \mathrm{wt} . \% \mathrm{EG}$ solutions is drawn in Figure $4 \mathrm{~b}$. An interesting feature of the $\mathrm{C} 12 \mathrm{PC}$ bilayer is the existence of an intermediate liquid crystalline $\left(\mathrm{L}_{\mathrm{x}}\right)$ phase, which is thought to be a nontilted, partially disordered $\mathrm{L}_{\alpha}$ phase, between the $\mathrm{P}_{\beta}{ }^{\prime}$ and $\mathrm{L}_{\alpha}$ phases. The $\mathrm{C} 12 \mathrm{PC}$ bilayer undergoes three kinds of transitions in EG solutions, in contrast with that in water, which undergoes only the main transition. Since the slope of the curve for the transition from the $\mathrm{L}_{\mathrm{c}}$ phase is smaller than that of the main transition curve, both curves cross each other at $40 \mathrm{MPa}$ on the diagram. We identified the former transition at pressures below $40 \mathrm{MPa}$ as the $\mathrm{L}_{\mathrm{c}} / \mathrm{L}_{\mathrm{x}}$ transition and above $40 \mathrm{MPa}$ as the $\mathrm{L}_{\mathrm{c}} / \mathrm{P}_{\beta}{ }^{\prime}$ transition. The higher-temperature transition in EG solution is the transition from the $\mathrm{L}_{\mathrm{x}}$ phase to the $\mathrm{L}_{\alpha}$ phase. The transition coincides with the main transition at about $180 \mathrm{MPa}$. The stability of the $\mathrm{L}_{\mathrm{x}}$ phase decreases gradually by pressure. At high pressures above $180 \mathrm{MPa}$, the $\mathrm{P}_{\beta}$ ' phase transforms into the $\mathrm{L}_{\alpha}$ phase directly.

\subsection{Asymmetry of Saturated Acyl Chain}

Most phospholipids contained in biological membranes of eukaryotic cells are not symmetric phospholipids with two equivalent acyl chains but asymmetric phospholipids with two different acyl chains [31]. We next describe the effect of the chain asymmetry on the phase behavior of PC bilayer membranes. Here we take up asymmetric saturated PCs with the chain difference of carbon number of 2 between the $s n-1$ and $s n-2$ chains, 1-palmitoyl-2-stearoyl-PC (PSPC) and its positional isomer 1-stearoyl-2-palmitoyl-PC (SPPC), 1-myristoyl-2-palmitoyl-PC (MPPC) and its positional isomer 1-palmitoyl-2-myristoyl-PC (PMPC). Two hydrophobic acyl chains in the $s n-1$ and $s n-2$ positions of symmetric PCs like DPPC are not originally equivalent with each other, although they have the chains of the same length. Since the sn-2 chain bends at the C2-C3 position in the molecule, the sn-2 chain becomes virtually 1.5 carbon-carbon bond lengths shorter at the end of the acyl chains than the $s n-1$ acyl chain if they take all-trans conformation in the gel phase [31,32]. Accordingly, the terminal methyl groups of both chains in the molecule are not aligned. In the case of PSPC (or MPPC) and SPPC (or PMPC), the distance at the terminal methyl ends between the $s n-1$ and $s n-2$ chains are 0.5 and 3.5 carbon-carbon lengths, respectively.

Figure 5 depicts the $T-p$ phase diagrams of the PSPC, SPPC, MPPC and PMPC bilayers [33-35]. The three phase transitions (sub-, pre- and main) can be observed in all bilayers in a manner similar to that of the DPPC bilayer, although the $\mathrm{L}_{\beta}{ }^{\prime}$ phase of the MPPC and PMPC bilayers turns to the metastable phase. All bilayers induce the $\mathrm{L}_{\beta} \mathrm{I}$ phase at a certain pressure irrespective of the chain asymmetry. The three transition temperatures of the PSPC or MPPC bilayer are larger than the corresponding bilayers of the positional isomer, SPPC or PMPC, respectively. This is because SPPC or PMPC with large chain asymmetry may reduce the stabilization of the $\mathrm{L}_{c}$ and gel phases in the bilayer because of the instability for a part of the chain difference between the $s n-1$ and $s n-2$ chains $[36,37]$. 
Figure 5. Temperature-pressure phase diagrams of bilayers of asymmetric $\mathrm{PCs}$ with saturated acyl chains: (a) PSPC, (b) SPPC, (c) MPPC, (d) PMPC. Phase transitions: (green) subtransition, (blue) pretransition and transitions between gel phases, (red) main transition. The gray-colored area in the figure of SPPC bilayer is the coexistence region between the $\mathrm{L}_{\beta}$ ' and $\mathrm{L}_{\beta} \mathrm{I}$ phases. Dashed line in each figure indicates the transition between metastable gel phases.
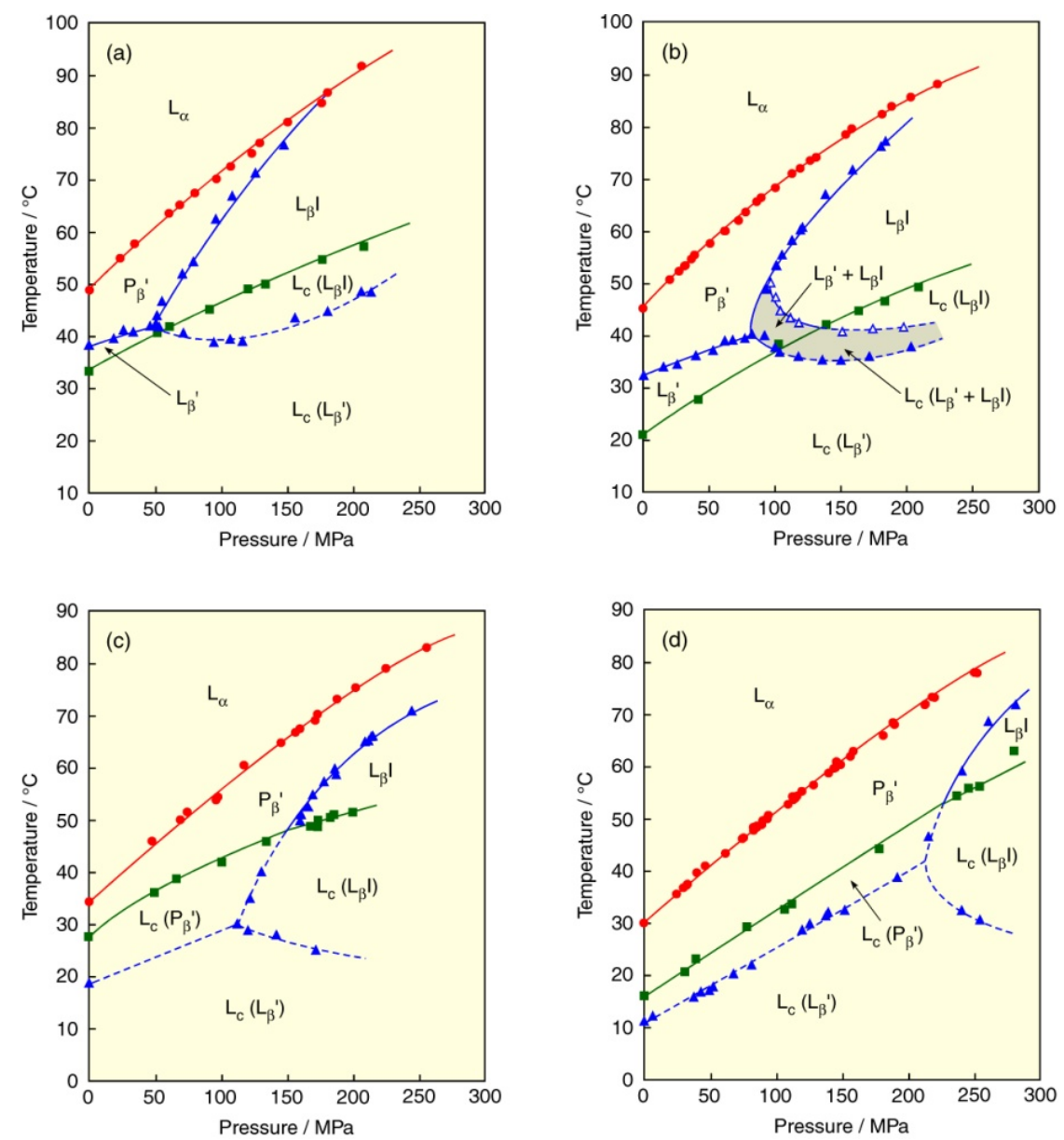

By contrast, in the PSPC or MPPC bilayer, the effect of the alignment of terminal methyl groups in acyl chains appears in the subtransition and the interdigitation: the alignment causes a significant elevation of the subtransition temperature and a marked depression of the MIP value. We can say that the membrane states with dense packing, like the $\mathrm{L}_{c}$ and $\mathrm{L}_{\beta} \mathrm{I}$ phases, definitely reflect the degree of the alignment of terminal methyl groups in acyl chains. In general, it is well known that the $\mathrm{L}_{c}$ phase formation of the bilayers of symmetric PCs with saturated acyl chains is very slow [26] and the formation requires a thermal treatment called annealing before experiments. On the other hand, the MPPC and PMPC can form the $\mathrm{L}_{c}$ phase easily on account of the metastability of the $\mathrm{L}_{\beta}$ ' phase, in particular, the $\mathrm{L}_{\mathrm{c}}$ phase formation of the MPPC bilayer is very fast and the fast formation enables us to study the state of the $\mathrm{L}_{c}$ phase in detail by a method of high-pressure fluorometry [38]. Moreover, there is a two-phase coexistence region (gray-colored area in the figure) of the $\mathrm{L}_{\beta}{ }^{\prime}$ and $\mathrm{L}_{\beta} \mathrm{I}$ phases in the SPPC bilayer. We elucidated from the high-pressure fluorometry [34,39] that the region results from a less polar "pocket" in the membrane, which is formed from the chain asymmetry in the terminal parts of acyl chains of SPPC. 


\subsection{Unsaturated Acyl Chain}

Biological membranes contain a high percentage of phospholipids with unsaturated fatty acids. For example, about half of the phospholipids in human erythrocyte membranes have unsaturated fatty acids such as oleic, linoleic, arachidonic and docosahexaenoic acids, etc. [3]. We next explain the effect of chain unsaturation on the phase behavior of PC bilayers. We take up two symmetric unsaturated PCs, dioleoyl-PC (DOPC) and dielaidoyl-PC (DEPC), which have two fatty acids with a carbon number of 18 and a double bond (cis or trans) at the C9-C10 position in each chain. The $T-p$ phase diagrams of the DOPC and DEPC bilayer membranes [40,41] are drawn in Figure 6a together with that of the bilayer of distearoyl-PC (DSPC: C18PC) that is a symmetric saturated homolog with the same carbon number. We can immediately notice that the bilayers of unsaturated PCs do not undergo the pretransition (transition between the gel phases) unlike the DSPC bilayer. This is because the acyl chains of unsaturated PCs become bulky in the presence of double bonds, which hinders the tilted orientation in the bilayer. Then, the DOPC and DEPC bilayers undergo phase transitions among the $\mathrm{L}_{c}, \mathrm{~L}_{\beta}$ and $\mathrm{L}_{\alpha}$ phases. We determined the transitions in reference to the results of experiments in 50 wt.\% EG solutions [40-42] whereby the DOPC bilayer undergoes the $\mathrm{L}_{\mathrm{c}} / \mathrm{L}_{\alpha}$ at low pressures or sub- $\left(\mathrm{L}_{c} / \mathrm{L}_{\beta}\right)$ transition at high pressures, and the main transition at all pressures, while the DEPC bilayer does the subtransition and the main transition at all pressures.

Figure 6. (a) Temperature-pressure phase diagrams of bilayers of symmetric PCs with unsaturated acyl chains: (1) DOPC, (2) DEPC, (3) DSPC (for comparison). (b) Temperature-pressure phase diagrams of bilayers of asymmetric PCs with an unsaturated acyl chains in the sn-2 position: (1) SOPC, (2) SLPC, (2)SAPC, (3) SDPC. Phase transitions: (green) subtransition or $\mathrm{L}_{\mathrm{c}} / \mathrm{L}_{\alpha}$ transition, (blue) pretransition and transitions between gel phases, (red) main transition. Dashed lines in figure (a) indicates the unstable main transition (curve 1) and the transition between metastable gel phases (curve 3).
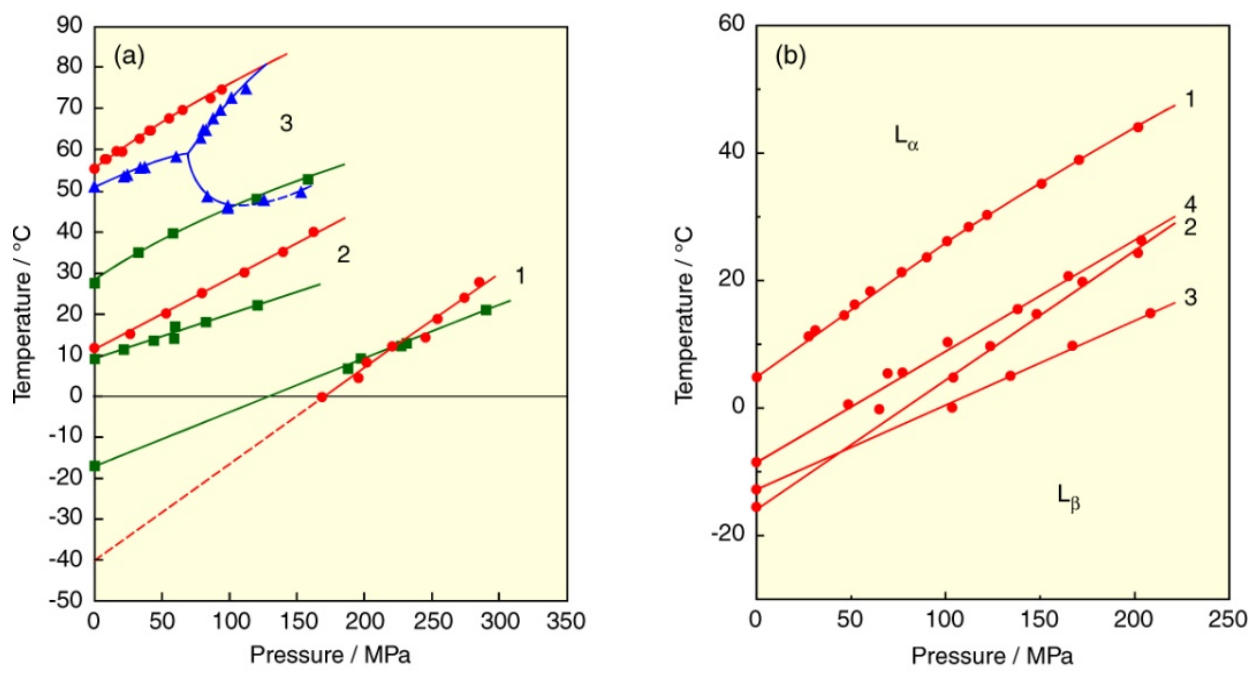

Comparing the main-transition temperatures of two kinds of unsaturated PC bilayer membranes under atmospheric pressure with that of the DSPC bilayer membrane, the temperatures are $55.6{ }^{\circ} \mathrm{C}$ for DSPC, $11.1{ }^{\circ} \mathrm{C}$ for DEPC and $-40.3{ }^{\circ} \mathrm{C}$ for DOPC. The substitution of two cis unsaturated chains for 
two saturated chains of DSPC produces the great depression of the main-transition temperature by about $48{ }^{\circ} \mathrm{C}$ per chain. The introduction of two trans double bonds to both chains is equivalent to almost half of the effect of two cis bonds. In the gel state of the cis unsaturated PC bilayers, the packing of the bilayer was disordered by the effect of the unsaturated chain because a cis unsaturation induced a bend of acyl chain. This leads to the reduction of van der Waals interaction between PC molecules and the gel phase is destabilized $[3,43]$. The destabilization of the gel phase by trans unsaturation is weaker than that by cis unsaturation because acyl chains with a trans double bond are relatively straight in the gel phase [44-46]. To keep high fluidity in biological membranes, it is essential that the membrane state is the $\mathrm{L}_{\alpha}$ phase. The great stabilization of the $\mathrm{L}_{\alpha}$ phase by introducing unsaturated fatty acids with a cis double bond to a lipid molecule is well correlated with the fact that membranes of living things in extreme conditions, such as deep sea, contain a high percentage of unsaturated phospholipids. A similar trend is observed for the bilayer transitions related to the $\mathrm{L}_{\mathrm{c}}$ phase under atmospheric pressure, that is, the subtransition temperatures are $28.2{ }^{\circ} \mathrm{C}$ for DSPC [26], $9.0{ }^{\circ} \mathrm{C}$ for DEPC and the $\mathrm{L}_{c} / \mathrm{L}_{\alpha}$ transition temperature is $-12.0{ }^{\circ} \mathrm{C}$ for DOPC. Unlike the main transition temperature, the temperature difference is not equivalent among all PC bilayers due to the difference in the kinds of the transition, this means that the structure of acyl chain also affects the stability of the $\mathrm{L}_{\mathrm{c}}$ phase and the effect is stronger in a cis double bond than a trans bond.

In the DOPC bilayer, the main-transition curve intersects the curve of the transition related to the $\mathrm{L}_{\mathrm{c}}$ phase at about $234 \mathrm{MPa}$, and the stability of gel $\left(\mathrm{L}_{\beta}\right)$ phase changes at the intersection point: the $\mathrm{L}_{\beta}$ phase changes from metastable to stable at the pressure. This means that the $\mathrm{L}_{\beta}$ phase at a low pressure becomes rather unstable, and only the $\mathrm{L}_{c} / \mathrm{L}_{\alpha}$ transition can be observed under atmospheric pressure [47]. Here great care should be taken in dealing with a phase transition of unsaturated phospholipids at a low temperature below the freezing point of water. The low-temperature phase transition of unsaturated phospholipid bilayers is generally regarded as the main (gel-liquid crystal) transition because only one transition is observed at a low temperature. However, at a temperature below the freezing point of water, the bilayers are prone to become the $\mathrm{L}_{c}$ phase because the bulk-water molecules freeze and simultaneously the molecules pull out the water molecules from the interlamellar region between the bilayers. And then, the $\mathrm{L}_{\beta}$ phase becomes metastable or unstable, and the observed phase transition is the $\mathrm{L}_{\mathrm{c}} / \mathrm{L}_{\alpha}$ transition, not the main transition as seen in the phase diagram of the DOPC bilayer. We have also examined the phase transitions of a series of $N$-methylated dioleoylphosphatidylethanolamine (DOPE) bilayers [42] and proved that the low-temperature phase transition of these DOPE bilayers is the $\mathrm{L}_{c} / \mathrm{L}_{\alpha}$ transition, although the transitions have been generally considered to be the main transition.

\subsection{Asymmetry of Unsaturated Mixed Acyl Chains}

As mentioned in the previous section, most phospholipids of biological membranes have asymmetric acyl chains. Especially, they have an unsaturated chain in either $s n-1$ or $s n-2$ position. A few reports have been made on phase transitions of PCs with mixed acyl chains of a saturated and an unsaturated one [48-50]. We selected six kinds of mixed-chain PCs with an unsaturated acyl chain in the $s n-1$ or $s n-2$ position, 1-oleoyl-2-stearoyl-PC (OSPC), 1-oleoyl-2-palmitoyl-PC (OPPC), 1-oleoyl-2-myristoyl-PC (OMPC) and 1-stearoyl-2-oleoyl-PC (SOPC), 1-palmitoyl-2-oleoyl-PC 
(POPC), 1-myristoyl-2-oleoyl-PC (MOPC). The corresponding $T-p$ phase diagrams of these mixed-chain PC bilayers are depicted in Figure 7 [40,41,51,52]. The bilayers of the mixed-chain PCs with an unsaturated acyl chain at the $s n-1$ position, OMPC, OPPC and OSPC, undergo two phase transitions, whereas those of the PCs with an unsaturated acyl chain at the $s n-2$ position, MOPC, POPC and SOPC, undergo one phase transition. In our previous studies [51,52], we identified from the thermal behavior and the pressure dependence of the phase-transition temperatures that the transitions observed in the OMPC and OPPC bilayers are the $\mathrm{L}_{c} / \mathrm{L}_{\alpha}$ or sub- $\left(\mathrm{L}_{c} / \mathrm{L}_{\beta}\right)$ transition and the main $\left(\mathrm{L}_{\beta} / \mathrm{L}_{\alpha}\right)$ transition and those observed in OSPC bilayer are the subtransition and the main transition, while the transition observed in MOPC, POPC and SOPC bilayers is the main transition. It is clear that the phase behavior of the bilayers of unsaturated mixed-chain PCs is greatly dependent on the binding position of an unsaturated acyl chain on the glycerol backbone.

Figure 7. (a) Temperature-pressure phase diagrams for bilayers of PCs with an unsaturated acyl chain in the $s n-1$ position: $(1 \mathrm{a}, 1 \mathrm{~b}) \operatorname{OSPC},(2 \mathrm{a}, 2 \mathrm{~b})$ OPPC and $(3 \mathrm{a}, 3 \mathrm{~b})$ OMPC. (b) Temperature-pressure phase diagrams for bilayers of PCs with an unsaturated acyl chain in the $s n-2$ position: (1) SOPC, (2) POPC, (3) MOPC. Open symbol refers to transitions in aqueous $50 \mathrm{wt}$ \% EG solution. Phase transitions: (green) $\mathrm{L}_{\mathrm{c}} / \mathrm{L}_{\beta}$ transition (1b) and $\mathrm{L}_{\mathrm{c}} / \mathrm{L}_{\alpha}$ or $\mathrm{L}_{\mathrm{c}} / \mathrm{L}_{\beta}$ transition $(2 \mathrm{~b}, 3 \mathrm{~b}$ ), (red) main transition. Dashed line in figure (a) indicates the unstable main transition (curve $3 \mathrm{a}$ ).
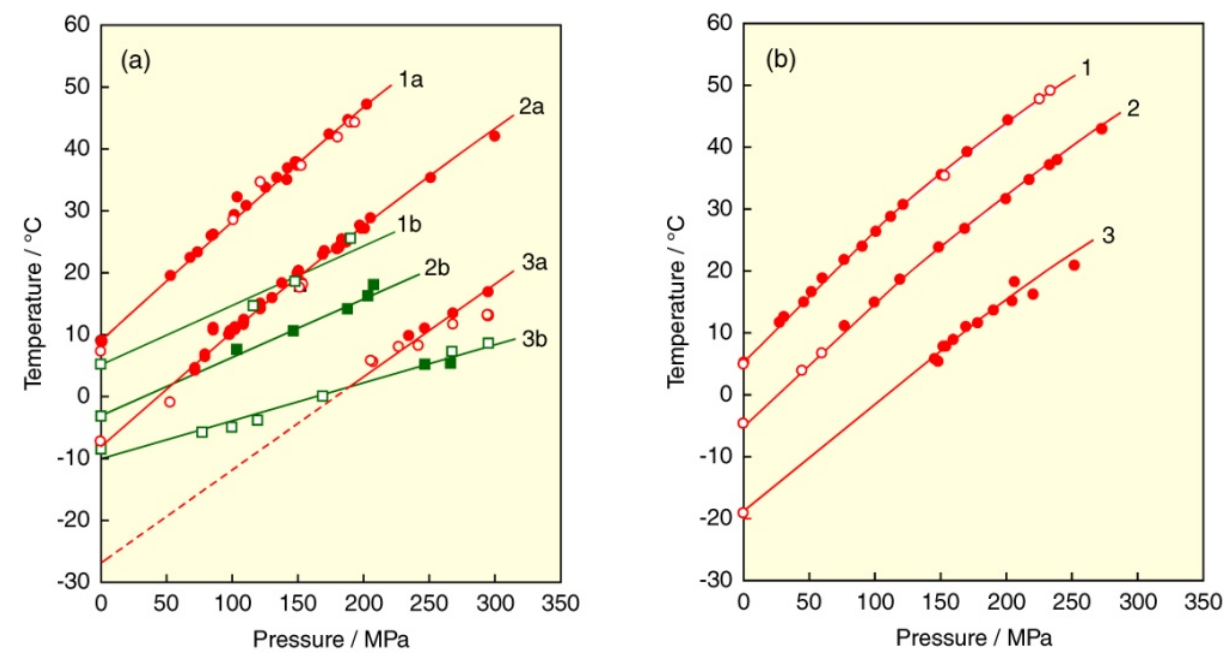

The phase-transition temperatures of the bilayers of all unsaturated mixed-chain PCs increase with applying pressure and also with an increase in chain length of saturated acyl chain in the $s n-1$ or $s n-2$ position. For the OMPC, OPPC and OSPC bilayers, the pressure dependence of the main-transition curve is larger than that of the curve of the transition related to the $\mathrm{L}_{c}$ phase. With a decrease in the chain length of saturated chains in the $s n-2$ position, the main-transition curve intersects the curve of the transition related to the $\mathrm{L}_{c}$ phase, and the stability of the $\mathrm{L}_{\beta}$ phase changes at the intersection point. Accordingly, while the $\mathrm{L}_{\beta}$ phase exists as a stable one in the whole range of pressure in the OSPC bilayer, the $\mathrm{L}_{\beta}$ phase changes from metastable to stable at $c a .50 \mathrm{MPa}$ in the OPPC bilayer and $c a .190$ $\mathrm{MPa}$ in the OMPC bilayer. In the case of the OMPC bilayer, since the $\mathrm{L}_{\beta}$ phase at a low pressure becomes unstable as the case of the DOPC bilayer [47], it is difficult to observe the main transition under atmospheric pressure. 
The main-transition temperatures of the OSPC and SOPC bilayers are $8.7{ }^{\circ} \mathrm{C}$ and $6.7{ }^{\circ} \mathrm{C}$, respectively. This means that the effect of the introduction of a cis double bond to the $s n-2$ chain is slightly larger than the effect of the introduction of that to the $s n-1$ chain [52]. It is known that most of naturally occurring asymmetric phospholipids are phospholipids with a saturated chain at the sn-1 position and an unsaturated chain at the $s n-2$ position. We must therefore consider the effect of the polyunsaturation in the $s n-2$ acyl chain on the bilayer phase behavior. The $T-p$ phase diagrams for the bilayers of SOPC, 1-stearoyl-2-linoleoyl-PC (SLPC), 1-stearoyl-2-arachidonoyl-PC (SAPC) and 1-stearoyl-2-docosahexaenoyl-PC (SDPC), which contain the stearate chain in the $s n-1$ position and the mono- or poly-unsaturated acyl chain in the $s n-2$ position, are shown in Figure 6b [53]. These unsaturated PC bilayers undergo the main transition under a normal condition. The transition temperatures of all bilayers increase linearly by applying pressure. The transition temperatures of the SOPC, SLPC, SAPC and SDPC bilayers under atmospheric pressure were 6.7, $-15.0,-13.0$ and $-9.0{ }^{\circ} \mathrm{C}$, respectively. The results suggest that the phase-transition temperature of unsaturated $\mathrm{PC}$ bilayers does not decrease monotonously with an increasing degree of unsaturation. Although the introduction of the first cis double bond into DSPC (yielding SOPC) lowers the phase transition temperature by $48.9{ }^{\circ} \mathrm{C}$ and two double bonds (SLPC) lowers it by a further $21.7{ }^{\circ} \mathrm{C}$, four and six double bonds (SAPC and SDPC) bring about no further decrease, and unexpectedly cause a slight increase in the transition temperature. We have thermodynamically elucidated from the smallest $\mathrm{d} T / \mathrm{d} p$ value of the SAPC bilayer that the SAPC bilayer has the gel phase with the loosest packing among these PC bilayers. On the other hand, the relatively high temperature of the main transition of the SDPC bilayer suggests that in the gel state, the bilayer exists in a rather regular and relatively stable conformation as compared to the other bilayers [54,55]. Since the acyl chain of SDPC must presumably be assumed to take a conformation approximately parallel to that of the bilayer normal, and motional freedom is severely limited by the lack of rotation at the six double bonds, allowed conformations are highly restricted. One of the possible conformations of SDPC in the gel state is as follows: the saturated $s n-1$ chain is well stretched, and the marked effect of the cis double bond in the $s n$-2 chain, including the reduction in the effective chain length, yields an approximately helical conformation because the hydrophobic chain of docosahexaenoic acid can take the conformation [56].

\section{Effect of Linkage of Glycerol Backbone on the Phase Behavior of PC Bilayer Membranes}

Diacyl-PCs are phospholipids, of which each of the two hydrophobic chains binds to the glycerol backbone by an ester linkage, and they are called ester-linked phospholipids. On the other hand, there exist other kinds of phospholipids, of which the hydrophobic chain binds to the glycerol backbone by an ether linkage, in the cell membranes of some organisms [57] and they are called ether-linked phospholipids. Plasmalogens (1-alkenyl-2-acyl-PC or -PE) in brain and myelin membranes and macrocyclic lipids with many ether-linked isoprenoid chains in archaebacteria and deep-sea microorganisms are typical examples of ether-linked phospholipids and their bilayer properties have been examined [58-61]. Next, we demonstrate the phase behavior of bilayers of dialkyl-PCs, which are PCs with two alkyl chains linking to the glycerol backbone by methylene groups $\left(-\mathrm{CH}_{2}-\right)$ instead of carbonyl groups $(>\mathrm{C}=\mathrm{O})$. The substitution of the linkage influences the bilayer phase behavior remarkably. For example, the bilayer of dihexadecyl-PC (DHPC) undergoes the 
pre- and main transitions with increasing temperature under atmospheric pressure like the DPPC bilayer. Although the main transition is the transition from the $\mathrm{P}_{\beta}{ }^{\prime}$ phase to the $\mathrm{L}_{\alpha}$ phase irrespective of the linkage difference, the pretransition of the DHPC bilayer is the transition from the $\mathrm{L}_{\beta} \mathrm{I}$ phase to the $\mathrm{P}_{\beta}^{\prime}$ phase [62-64], and not the transition from the $\mathrm{L}_{\beta}{ }^{\prime}$ phase as observed in the DPPC bilayer. The DHPC bilayer induces the interdigitation by only hydration under atmospheric pressure because it weakens the interaction between adjacent lipid molecules. Since the pre- and main-transition temperatures of the DHPC and DPPC bilayers under atmospheric pressure are very close to each other, and both bilayers give similar thermodynamic quantities of the transition, it is difficult to distinguish the phase states by a method as DSC. In contrast to this, the phase states are definitely distinguished by pressurization of the both bilayers.

Figure $8 \mathrm{a}$ illustrates the $T-p$ phase diagrams constructed for the bilayers of four kinds of dialkyl-PCs, didodecyl-PC (O-C12PC), ditetradecyl-PC $(O-\mathrm{C} 14 \mathrm{PC})$, dihexadecyl-PC (O-C16PC (DHPC)) and dioctadecyl-PC $(O-\mathrm{C} 18 \mathrm{PC})$, respectively $[65,66]$. The difference in the pretransition between dialkyl- and diacyl-PCs appears in the slope of the pretransition curve on the phase diagrams: the slopes of the transition $\left(\mathrm{L}_{\beta}{ }^{\prime} / \mathrm{P}_{\beta}{ }^{\prime}\right.$ transition) for the diacyl-PC bilayers are smaller than those of the main-transition curve, while those of the transition $\left(\mathrm{L}_{\beta} \mathrm{I} / \mathrm{P}_{\beta}{ }^{\prime}\right.$ transition) for the dialkyl-PC bilayers are conversely larger than them. Therefore, the pretransition and main transition curves intersect with each other at a certain pressure, then the $\mathrm{P}_{\beta}$ ' phase disappears at high pressures above the intersection point and only the $\mathrm{L}_{\beta} \mathrm{I} / \mathrm{L}_{\alpha}$ transition occurs in the high-pressure region. This means that pressure stabilizes the $\mathrm{L}_{\beta} \mathrm{I}$ phase but destabilizes the $\mathrm{P}_{\beta}{ }^{\prime}$ and $\mathrm{L}_{\alpha}$ phases. The effect of the alkyl chain length on the phase behavior synergizes the pressure effect, that is, the effect further extends the region of the $\mathrm{L}_{\beta} \mathrm{I}$ phase, while further shrinking the region of the $\mathrm{P}_{\beta}{ }^{\prime}$ and $\mathrm{L}_{\alpha}$ phases. In addition, there is no pressure-induced phase in all the dialkyl-PC bilayers investigated.

Figure 8. (a) Temperature-pressure phase diagrams of dialkyl-PC (ether-linked $\mathrm{PC}$ ) bilayers: (1) $O-\mathrm{C} 12 \mathrm{PC}$, (2) $O-\mathrm{C} 14 \mathrm{PC}$, (3) $O-\mathrm{C} 16 \mathrm{PC}$ (DHPC), (4) $O-\mathrm{C} 18 \mathrm{PC}$. Phase transitions: (blue) pre- $\left(\mathrm{L}_{\beta} \mathrm{I} / \mathrm{P}_{\beta}{ }^{\prime}\right)$ transition, (red) main transition. (b) Comparison of temperature-pressure phase diagram of DHPC bilayer (upper right diagram) with that of DPPC bilayer (lower left dagram).
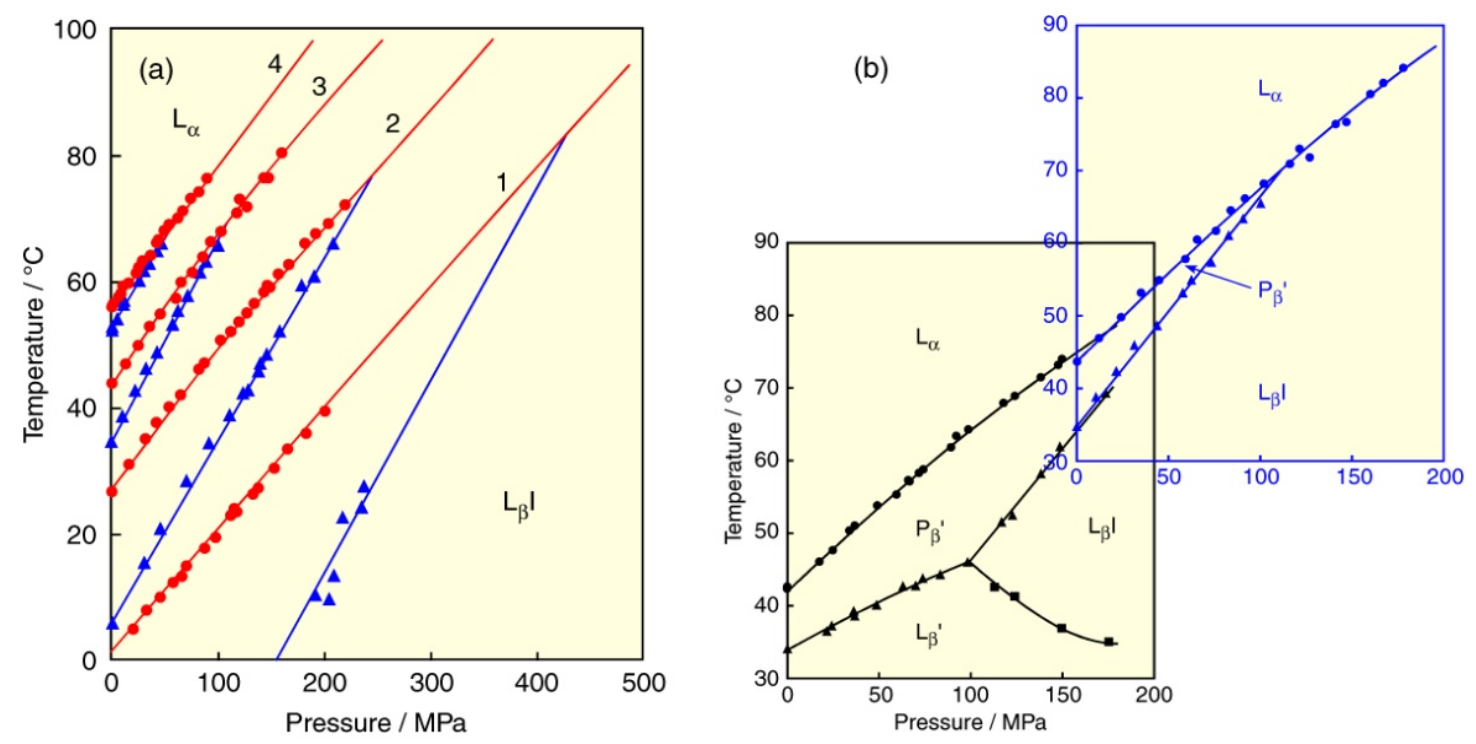
The $T-p$ phase diagrams of the dialkyl-PC bilayers are quite different from those of the diacyl-PC bilayers given in Figures 2 and 3. The phase diagrams of the ether-linked PC bilayers are much simpler than the corresponding ester-linked PC bilayers [21] because of the lack of a pressure-induced phase. The substitution of an ether linkage for an ester linkage brings about the appearance of the $\mathrm{L}_{\beta} \mathrm{I}$ phase under ambient pressure. Overlapping the phase diagram of the DHPC bilayer in Figure 8a with that of the DPPC bilayer in Figure 2, we immediately notice that the phase behavior of the DPPC bilayer in a high-temperature and pressure region corresponds to the DHPC bilayers in the normal temperature and pressure region (Figure $8 \mathrm{~b}$ ). Furthermore, the overlap of the phase diagrams between both PC bilayers extends to the lower pressure region with an increase in hydrophobic chain length (data not shown, see ref. [66]), namely the elongation of hydrophobic chain length brings the phase behavior of both PC bilayers into closer relationship. When considering that membrane lipids of archaebacteria and deep-sea microorganisms are almost all ether-linked phospholipids and that an ether-linkage is chemically more stable than an ester linkage, it is so interesting that the phase behavior of the ether-linked PC bilayers bears a strong resemblance to that of the ester-linked PC bilayers under such extreme conditions as high temperature and pressure. The phase behavior of the dialkyl-PC bilayers means that the bilayers become more rigid than the diacyl-PC bilayer, and this behavior seems to be inconsistent with the fact at a glance that organisms living in an extreme condition have softer membranes. We speculate that the linkage-type change from the chemically stable ether-linkage at a high temperature and pressure to the chemically degradable ester-linkage is due to the environmental adaptation in the organic evolutionary process. However, a recent study on biological membranes of deep-sea microorganisms under high pressure [67] has shown that the membranes are not necessarily softer than those under atmospheric pressure, and do have, in fact, a rather rigid structure.

\section{Effect of Solvent on the Phase Behavior of DPPC Bilayer Membrane}

Finally, we describe the effect of a solvent on the PC bilayer. In Section 2, we mentioned the lyotropic phase transitions of the DPPC bilayer induced by hydration. Here we show how the difference in hydration force of a solvent affects the phase behavior of the DPPC bilayer. For this purpose, we used two solvents, one is heavy water $\left(\mathrm{D}_{2} \mathrm{O}\right)$ with stronger hydration force than light water $\left(\mathrm{H}_{2} \mathrm{O}\right)$, the other is an ethanol $(\mathrm{EtOH})$ solution with weaker hydration force than $\mathrm{H}_{2} \mathrm{O}$. Several kinds of studies with respect to solvent effects on the DPPC bilayer have been reported [68-71]. The constructed $T-p$ phase diagrams of the DPPC bilayer in $\mathrm{D}_{2} \mathrm{O}$ and in $0.4 \mathrm{M}$ ethanol solution are shown in Figure 9 in comparison with the diagram in $\mathrm{H}_{2} \mathrm{O}$, respectively $[72,73]$. The main $\left(\mathrm{P}_{\beta}{ }^{\prime} / \mathrm{L}_{\alpha}\right)$ transition in $\mathrm{D}_{2} \mathrm{O}$ is almost consistent with that in $\mathrm{H}_{2} \mathrm{O}$ while it moves to the lower-temperature region to some extent at constant pressure in an $\mathrm{EtOH}$ solution than in $\mathrm{H}_{2} \mathrm{O}$. In contrast to the behavior of the main transition, the transitions between the gel phases (pre- $\left(\mathrm{L}_{\beta}{ }^{\prime} / \mathrm{P}_{\beta}{ }^{\prime}\right), \mathrm{L}_{\beta}{ }^{\prime} / \mathrm{L}_{\beta} \mathrm{I}$ and $\mathrm{L}_{\beta} \mathrm{I} / \mathrm{P}_{\beta}{ }^{\prime}$ ) are markedly influenced by the substitution of solvent. This suggests that the substitution of solvent is more effective for the region of bilayer/water interface than for the hydrophobic core part of the bilayer. 
Figure 9. (a) Temperature-pressure phase diagrams of DPPC bilayer: (blue) in $\mathrm{D}_{2} \mathrm{O}$, (black) in $\mathrm{H}_{2} \mathrm{O}$. (b) Temperature-pressure phase diagrams of DPPC bilayer: (red) in $0.4 \mathrm{M}$ ethanol solution, (black) in $\mathrm{H}_{2} \mathrm{O}$. Phase transitions are the same as those in Figure $2 \mathrm{~b}$.
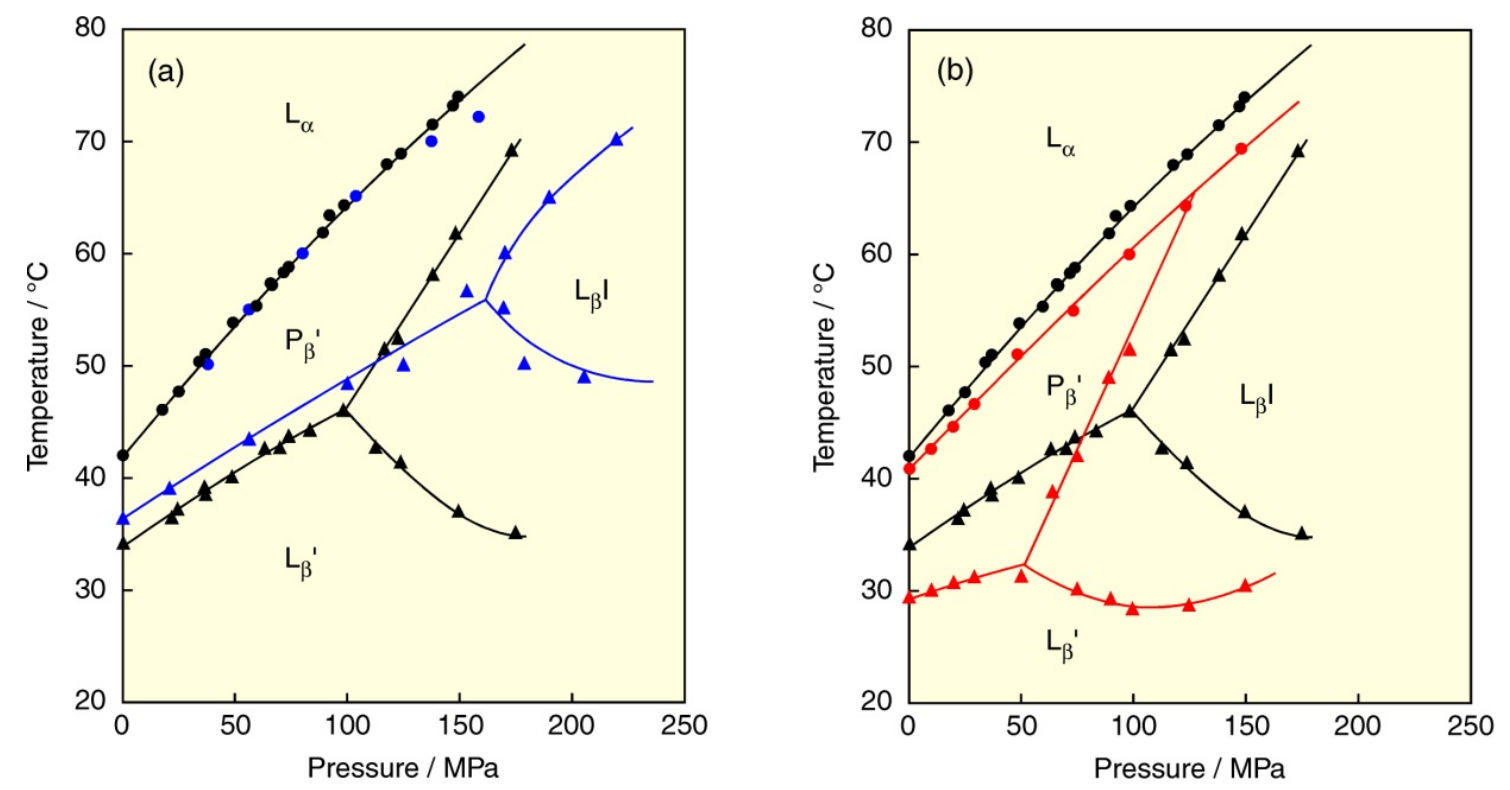

By substituting $\mathrm{D}_{2} \mathrm{O}$ for $\mathrm{H}_{2} \mathrm{O}$, the $\mathrm{L}_{\beta}{ }^{\prime} / \mathrm{P}_{\beta}^{\prime}$ and $\mathrm{L}_{\beta}{ }^{\prime} / \mathrm{L}_{\beta} \mathrm{I}$ transition temperatures elevate, while the $\mathrm{L}_{\beta} \mathrm{I} / \mathrm{P}_{\beta}{ }^{\prime}$ transition temperature depresses. These temperature changes can be explained as follows. The hydrogen bonds are stronger in $\mathrm{D}_{2} \mathrm{O}$ than in $\mathrm{H}_{2} \mathrm{O}$ [74,75], and this strong solvent interaction reduces an optimal area of the DPPC molecule in the bilayer. Because the area of the DPPC molecule in the gel phases increases in the order of the $\mathrm{L}_{\beta^{\prime}}\left(0.39 \mathrm{~nm}^{2}\right), \mathrm{P}_{\beta}{ }^{\prime}\left(0.41 \mathrm{~nm}^{2}\right)$ and $\mathrm{L}_{\beta} \mathrm{I}\left(0.78 \mathrm{~nm}^{2}\right)$ phases $[63,76]$, the stabilization of the gel phases in $\mathrm{D}_{2} \mathrm{O}$ in comparison with those in $\mathrm{H}_{2} \mathrm{O}$ is enhanced in the reverse order: $\mathrm{L}_{\beta}{ }^{\prime}>\mathrm{P}_{\beta}{ }^{\prime}>\mathrm{L}_{\beta} \mathrm{I}$. The difference in stabilization among the gel phases results in the temperature elevation of the transition from left to right in the above inequality of the gel-phase stabilization, while it results in the temperature depression of the transition from right to left in the inequality. The change in stability between the gel phases by $\mathrm{D}_{2} \mathrm{O}$ produces the destabilization of the $\mathrm{L}_{\beta} \mathrm{I}$ phase and the region of the $\mathrm{L}_{\beta} \mathrm{I}$ phase moves to the high temperature and pressure region. Hence $\mathrm{D}_{2} \mathrm{O}$ suppresses the formation of the $\mathrm{L}_{\beta} \mathrm{I}$ phase. The bilayer interdigitation of phospholipid bilayers under high pressure was first observed for the DPPC bilayer in $\mathrm{D}_{2} \mathrm{O}$ by a method of small angle neutron scattering [12]; however, the effect of $\mathrm{D}_{2} \mathrm{O}$ on the phase behavior was not at all mentioned. We were later able to clarify the effect [72].

By contrast, the substitution of an EtOH solution for $\mathrm{H}_{2} \mathrm{O}$ causes a contrary situation: the $\mathrm{L}_{\beta}{ }^{\prime} / \mathrm{P}_{\beta}{ }^{\prime}-$ and $\mathrm{L}_{\beta}{ }^{\prime} / \mathrm{L}_{\beta} \mathrm{I}$-transition temperatures are depressed, while the $\mathrm{L}_{\beta} \mathrm{I} / \mathrm{P}_{\beta}{ }^{\prime}$-transition temperature elevates. Since EtOH decreases the hydrogen bonding in the solution, the optimal area of the DPPC molecule in the bilayer increases. Then the stabilization of the gel phases in an EtOH solution in comparison with those in $\mathrm{H}_{2} \mathrm{O}$ is enhanced in the same order as the area in the gel phase: $\mathrm{L}_{\beta}{ }^{\prime}<\mathrm{P}_{\beta}{ }^{\prime}<\mathrm{L}_{\beta} \mathrm{I}$, which brings about the opposite effect on the transition temperatures between the gel phases compared to $\mathrm{D}_{2} \mathrm{O}$. Consequently, an EtOH solution promotes the formation of the $\mathrm{L}_{\beta} \mathrm{I}$ phase $[68,73]$, that is, the $\mathrm{L}_{\beta} \mathrm{I}$ phase is stabilized and the region of the $\mathrm{L}_{\beta} \mathrm{I}$ phase moves to a low temperature and pressure region. The depression in the main-transition temperature in an EtOH solution compared with in $\mathrm{D}_{2} \mathrm{O}$ or in $\mathrm{H}_{2} \mathrm{O}$ 
suggests that a slight amount of EtOH molecules are incorporated into the hydrophobic core part of the bilayer and suppresses the chain melting.

\section{Conclusions}

In this review, we first described the phase behavior of the DPPC bilayer. We subsequently explained the various kinds of PCs with different molecular structures from DPPC by using the $T-p$ phase diagrams, and finally illustrated the solvent effect on the phase behavior. To summarize the phase behavior of the PC bilayers, several phase states that the PC bilayers show are common to all PC bilayers, that is, the $\mathrm{L}_{c}, \mathrm{~L}_{\beta}$ and $\mathrm{L}_{\alpha}$ phases. It is characteristic of PCs with saturated acyl chains to exhibit the polymorphism of the gel phases such as $\mathrm{L}_{\beta}{ }^{\prime}, \mathrm{P}_{\beta}{ }^{\prime}$ and $\mathrm{L}_{\beta} \mathrm{I}$ phases, due to the steric hindrance between adjacent choline head groups of the PC molecules in the bilayer. The polymorphism is markedly affected by the chain length, chain asymmetry, linkage type and solvent substitution. It is also interesting that ester-linked PCs (diacyl-PCs) with an acyl-chain length of a carbon number between 14 and 21 induce the $\mathrm{L}_{\beta} \mathrm{I}$ phase under high pressure, while ether-linked PCs (dialkyl-PCs) induce the $\mathrm{L}_{\beta} \mathrm{I}$ phase under atmospheric pressure by only hydration. On the other hand, the introduction of double bonds into acyl chains produces the great stabilization of the $\mathrm{L}_{\alpha}$ phase, and the effect is also markedly affected by the kind (cis or trans) and number of a double bond and the position of the unsaturated chain ( $s n-1$ or $s n-2)$. Consequently, we can say that the PC bilayers undergo various kinds of phase transitions, depending on hydration, temperature, pressure and solvent, and even a slight difference in the molecule structure of the PC molecule will yield a significant influence on the bilayer phase behavior.

\section{Acknowledgments}

The authors are very grateful to Shoji Kaneshina, professor emeritus of The University of Tokushima, for his kind help and advice in discussion of lipid phase behavior under high pressure.

\section{References}

1. Yeagle, P.L. The Structure of Biological Membranes, 2nd ed.; CRC Press: New York, NY, USA, 2005.

2. Dopico, A.M. Methods in Membrane Lipids (Methods in Molecular Biology 400); Humana Press: Totowa, NJ, USA, 2007.

3. Heimberg, T. Thermal Biophysics of Membranes; Wiley-VCH: Weiheim, Germany, 2007.

4. Caffrey, M. LIPIDAT, A Database of Thermodynamic Data and Associated Information on Lipid Mesomorphic and Polymorphic Transitions; CRC Press: Boca Raton, FL, USA, 1993.

5. Wilkinson, D.A.; Nagle, J.F. Thermodynamics of Lipid Bilayers. In Liposomes: from Physical Structure to Therapeutic Applications; Kninght, C.G., Ed.; Elsevier: Amsterdam, The Netherlands, 1981; pp. 273-297.

6. Koynova, R.; Caffrey, M. Phases and phase transitions of the phosphatidylcholines. Biochim. Biophys. Acta 1998, 1376, 91-145. 
7. Nagle, J.F.; Tristram-Nagle, S. Structure of lipid bilayers. Biochim. Biophys. Acta 2000, 1469, 159-195.

8. Trudell, J.R.; Payan, D.G.; Chin, J.H.; Cohen, E.N. Pressure-induced elevation of phase transition temperature in dipalmitoylphosphatidylcholine bilayers. An electron spin resonance measurement of the enthalpy of phase transiton. Biochim. Biophys. Acta 1974, 373, 436-443.

9. Srinivasan, K.R.; Kay, R.L.; Nagle, J.F. The pressure dependence of the lipid bilayer phase transition. Biochemistry 1974, 13, 3494-3496.

10. Chong, P.L.G.; Weber, G. Pressure dependence of 1,6-diphenyl-1,3,5-hexatrien fluorescence in single-component phosphatidylcholine liposomes. Biochemistry 1983, 22, 5544-5550.

11. Wong, P.T.T.; Mantsch, H.H. Effects of hydrostatic pressure on the molecular structure and endothermic phase transitions of phosphatidylcholine bilayers: A Raman scattering study. Biochemistry 1985, 24, 4091-4096.

12. Bragnza, L.F.; Worcester, D.L. Hydrostatic pressure induces hydrocarbon chain interdigitation in single component phospholipid bilayers. Biochemistry 1986, 25, 2591-2596.

13. Prasad, S.K.; Shashidhar, R.; Gaber, B.P.; Chandrasekhar, S.C. Pressure studies on two hydrated phospholipids-1,2-dimristoyl-phosphatidylcholine and 1,2-dipalmitoyl-phosphatidylcholine. Chem. Phys. Lipids 1987, 43, 227-235.

14. Winter, R.; Pilgrim, W.C. A SANS study of high pressure phase transitions in model biomembranes. Ber. Bunsenges. Phys. Chem. 1989, 93, 708-717.

15. Driscoll, D.A.; Samarasinghe, J.; Adamy, S.; Jonas, J.; Jonas, A. Pressure effects on dipalmitoylphosphatidylcholine bilayers measured by ${ }^{2} \mathrm{H}$ nuclear magnetic resonance. Biochemistry 1991, 30, 3322-3327.

16. Hauser, H.; Pascher, I.; Pearson, R.H.; Sundell, S. Preferred conformation and molecular packing of phosphatidylethanolamine and phosphatidylcholine. Biochim. Biophys. Acta 1981, 650, 21-51.

17. Kodama, M.; Kato, H.; Aoki, H. Comparison of differently bound molecules in the gel and subgel phases of a phospholipids bilyaer system. J. Therm. Anal. Cal. 2001, 64, 219-230.

18. Kodama, M.; Kuwabara, M.; Seki, S. Successive phase-transition phenomena and phase diagram of the phosphatidylcholine-water system as revealed by differential scanning calorimetry. Biochim. Biophys. Acta 1982, 689, 567-570.

19. Kodama, M. Phase transition phenomena induced by the successive appearances of new types of aggregation states of water molecules in the "L-dipalmitoylphosphatidylcholine-water" system. Thermchim. Acta 1986, 109, 81-89.

20. Kusube, M.; Matsuki, H.; Kaneshina, S. Thermotropic and barotropic phase transitions of $\mathrm{N}$-methylated dipalmitoylphosphatidylethanolamine bilayers. Biochim. Biophys. Acta 2005, 1668, $25-32$.

21. Ichimori, H.; Hata, T.; Matsuki, H.; Kaneshina, S. Baroropic phase transitions and pressure-induced interdigitation on bilayer membranes of phospholipids with varying acyl chain-lengths. Biochim. Biophys. Acta 1998, 1414, 165-174.

22. Ichimori, H.; Hata, T.; Yoshioka, T.; Matsuki, H.; Kaneshina, S. Thermotropic and barotropic phase transition on bilayer membranes of phospholipids with varying acyl chain-lengths. Chem. Phys. Lipids 1997, 89, 97-105. 
23. Nagle, J.F.; Wilkinson, D.A. Density measurements and molecular interactions. Biophys. J. 1978, $23,159-175$.

24. Blume, A. Apparent molar heat capacities of phospholipids in aqueous dispersion. Effects of chain length and head group structure. Biochemistry 1983, 22, 5436-5442.

25. Stümpel, J.; Eibl, H.; Nichsch, A. X-ray analysis and calorimetry on phosphatidylcholine model membranes. The influence of length and position of acyl chains upon structure and phase behaviour. Biochim. Biophys. Acta 1983, 727, 246-254.

26. Lewis, R.N.A.H.; Mak, N.; McElhaney, R.N. A differential scanning calorimetric study of the thermotropic phase behavior of model membranes composed of phosphatidylcholines containing linear saturated fatty acyl chains. Biochemistry 1987, 26, 6118-6126.

27. Goto, M.; Wilk, A.; Kazama, A.; Chodankar, S.; Kohlbrecher, J.; Matsuki, H. Chain elongation of diacylphosphatidylcholine induces fully bilayer interdigitation under atmospheric pressure. Colloids Surf. B 2011, 84, 44-48.

28. Finegold, L.; Shaw, W.A.; Singer M.A. Unusual phase properties of dilauryl phosphatidylcholine (C12PC). Chem. Phys. Lipids 1990, 53, 177-184.

29. Hatta, I.; Matsuoka, S.; Singer, M.A.; Finegold, L. A new liquid crystalline phase in phosphatidylcholine bilayers as studied by X-ray diffraction. Chem. Phys. Lipids 1994, 69, 129-136.

30. Tada, K.; Goto, M.; Tamai, N.; Matsuki, H.; Kaneshina, S. Thermotropic and barotropic phase transitions of dilauroylphosphatidylcholine bilayer. Chem. Phys. Lipids 2008, 153, 138-143.

31. Huang, C.; Mason, J.T. Structure and properties of mixed-chain phospholipid assemblies. Biochim. Biophys. Acta 1986, 864, 423-470.

32. Bultmann, T.; Lin, H.; Wang, Z.; Huang, C. Thermotropic and mixing behavior of mixed-chain phosphatidylcholines with molecular weights identical with that of L- $\alpha$-dipalmitoylphosphatidylcholine. Biochemistry 1991, 30, 7194-7202.

33. Matsuki, H.; Goto, M.; Kusube, M.; Tamai, N.; Kaneshina, S. Barotropic phase transitions of 1-palmitoyl-2-stearoylphosphatidylcholine bilayer membrane. Chem. Lett. 2005, 34, 270-271.

34. Goto, M.; Kusube, M.; Tamai, N.; Matsuki, H.; Kaneshina, S. Effect of hydrostatic pressure on the bilayer phase behavior of symmetric and asymmetric phospholipids with the same total chain length. Biochim. Biophys. Acta 2008, 1778, 1067-1078.

35. Goto, M.; Ishida, S.; Tamai, N.; Matsuki, H.; Kaneshina, S. Chain asymmetry alters thermotropic and barotropic properties of phospholipid bilayer membranes. Chem. Phys. Lipids 2009, 161, $65-76$.

36. Stümpel, J.; Nichsch, A.; Eibl, H. Calorimetric studies on saturated mixed-chain lecithin-water systems. Nonequivalence of acyl chains in the thermotropic phase transition. Biochemistry 1981, 20, 662-665.

37. Lin, H.; Wang, Z.; Huang, C. The influence of acyl chain-length asymmetry on the phase transition parameters of phosphatidylcholine dispersions. Biochim. Biophys. Acta 1991, 1067, $17-28$.

38. Goto, M.; Wilk, A.; Kataoka, K.; Chodankar, S.; Tamai, N.; Fukui, M.; Kohlbrecher, J.; Ito, H.; Matsuki, H. Study on the subgel-phase formation using an asymmetric phospholipidb membrane by high-pressure fluorometry. Langmuir 2012, 28, 12191-12198. 
39. Goto, M.; Matsui, T.; Tamai, N.; Matsuki, H.; Kaneshina, S. Prodan fluorescence detects the bilayer packing of asymmetric phospholipids. Colloids Surf. B 2011, 84, 55-62.

40. Kaneshina, S.; Ichimori, H.; Hata, T.; Matsuki, H. Barotropic phase transitions of dioleoylphosphatidylcholine and stearoyl-oleoylphosphatidylcholine bilayer membranes. Biochim. Biophys. Acta 1998, 1374, 1-8.

41. Ichimori, H.; Hata, T.; Matsuki, H.; Kaneshina, S. Effect of unsaturated acyl chains on the thermotropic and barotropic phase transitions of phospholipid bilayer membranes. Chem. Phys. Lipids 1999, 100, 151-154.

42. Kusube, M.; Goto, M.; Tamai, N.; Matsuki, H.; Kaneshina, S. Bilayer phase transitions of $N$-methylated dioleoylphosphatidylethanolamines under high pressure. Chem. Phys. Lipids 2006, 142, 94-102.

43. Lewis, R.N.A.H.; McElhaney, R.N. The Mesomorphic Phase Behavior of Lipid Bilayers. In The Structure of Biological Membranes, 2nd ed.; Yeagle, P.L., Ed.; CRC Press: New York, NY, USA, 2005; pp. 66-69.

44. Silvius, J.R.; McElhaney, R.N. Effects of phospholipid acyl chain structure on thermotropic phase properties. 2: Phosphatidylcholines with unsaturated or cyclopropane acyl chains. Chem. Phys. Lipids 1979, 25, 125-134.

45. Seelig, J.; Waespe-Sarcevic, N. Molecular order in cis and trans unsaturated phospholipid bilayers. Biochemistry 1978, 17, 3310-3315.

46. Siminovitch, D.J.; Wong, P.T.T.; Mantsch, H.H. Effects of cis and trans unsaturation on the structure of phospholipid bilayer: A high-pressure infrared spectroscopic study. Biochemistry 1987, 26, 3277-3287.

47. Lewis, R.N.A.H.; Sykes, B.D.; McElhaney, R.N. Thermotropic phase behavior of model membranes composed of phosphatidylcholines containing cis-monounsaturated acyl chain homologues of oleic acid: Differential scanning calorimetric and ${ }^{31} \mathrm{P}$ NMR spectroscopic studies. Biochemistry 1998, 27, 880-887.

48. Davis, P.J.; Fleming, B.D.; Coolbear, K.P.; Keough, K.M.W. Gel to liquid crystalline transition temperatures of water dispersions of two pairs of positional isomers of unsaturated mixed-acid phosphatidylcholines, Biochemistry 1981, 20, 3633-3636.

49. Coolbear, K.P.; Berde, C.B.; Keough, K.M. Gel to liquid-crystalline phase transitions of aqueous dispersions of polyunsaturated mixed-acid phosphatidylcholines. Biochemistry 1983, 22, 1466-1473.

50. Keough, K.M.W.; Griffin, B.; Kariel, N. The influence of unsaturation on the phase transition temperatures of a series of heteroacid phosphatidylcholines containing twenty-carbon chains. Biochim. Biophys. Acta 1987, 902, 1-10.

51. Tada, K.; Saito, K.; Goto, M.; Tamai, N.; Matsuki, H.; Kaneshina, S. High-pressure study on bilayer phase behavior of oleoylmyristoyl- and myristoyloleoyl-phosphatidylcholines. Biophys. Chem. 2008, 138, 36-41.

52. Tada, K.; Miyazaki, E.; Goto, M.; Tamai, N.; Matsuki, H.; Kaneshina, S. Barotropic and thermotropic bilayer phase behavior of positional isomers of unsaturated mixed-chain phosphatidylcholines. Biochim. Biophys. Acta 2009, 1788, 1056-1063. 
53. Tada, K.; Goto, M.; Tamai, N.; Matsuki, H.; Kaneshina, S. Pressure effect on the bilayer phase transition of asymmetric lipids with an unsaturated acyl chain. Ann. N.Y. Acad. Sci. 2010, 1189, $77-85$.

54. Holte, L.L.; Separovic, F.; Gawrisch, K. Nuclear magnetic resonance investigation of hydrocarbon chain packing in bilayers of polyunsaturated phospholipids. Lipids 1996, 31, 199-203.

55. Koenig, B.W.; Strey, H.H.; Gawrisch, K. Membrane lateral compressibility determined by NMR and X-ray diffraction: Effect of acyl chain polyunsaturation. Biophys. J. 1997, 73, 1954-1966.

56. Applegate, K.R.; Glomset, T.A. Computer-based modeling of the conformation and packing properties of docosahexaenoic acid. J. Lipid Res. 1986, 27, 658-680.

57. Paltauf, F. Ether lipids in biomembranes. Chem. Phys. Lipids 1994, 74, 101-139.

58. Han, X.; Gross, R.W. Plasmenylcholine and phosphatidylcholine membrane bilayers possess distinct conformational motifs. Biochemistry 1990, 29, 4992-4996.

59. Lohner, K. Is the high propensity of ethanolamine plasmalogens to form non-lamellar lipid structures manifested in the properties of biomembranes? Chem. Phys. Lipids 1996, 81, 167-184.

60. Broniec, A.; Goto, M.; Matsuki, H. A peculiar phase transition of plasmalogen bilayer membrane under high pressure. Langmuir 2009, 25, 11265-11268.

61. Chong, P.L.G. Archaebacterial bipolar tetraether lipids; physico-chemical and membrane properties. Chem. Phys. Lipids 2010, 163, 253-265.

62. Ruocco, M.J.; Siminovitch, D.J.; Griffin, R.G. Comparative study of the gel phases of ether- and ester-linked phosphatidylcholines. Biochemistry 1985, 24, 2406-2411.

63. Laggner, P.; Lohner, K.; Degovics, G.; Müller, K.; Schuster, A. Structure and thermodynamics of the dihexadecylphosphatidylcholine-water system. Chem. Phys. Lipids 1987, 44, 31-60.

64. Kim, J.T.; Mattai, J.; Shipley, G.G. Gel phase polymorphism in ether-linked dihexadecylphosphatidylcholine bilayers. Biochemstry 1987, 26, 6592-6598.

65. Maruyama, S.; Matsuki, H.; Ichimori, I.; Kaneshina, S. Thermotropic and barotropic phase behavior of dihexadecylphosphatidylcholine bilayer membrane. Chem. Phys. Lipids 1996, 82, 125-132.

66. Matsuki, H.; Miyazaki, E.; Sakano, F.; Tamai, N,; Kaneshina, S. Thermotropic and barotropic phase transitions in bilayer membranes of ether-linked phospholipids with varying alkyl chain lengths. Biochim. Biophys. Acta 2007, 1768, 479-489.

67. Usui, K.; Hiraki, T.; Kawamoto, J.; Kurihara, T.; Nogi, Y.; Abe F. Eicosapentaenoic acid plays a role in stabilizing dynamic membrane structure in the deep-sea piezophile Shewanella violacea: A study employing high-pressure time-resolved fluorescence anisotropy measurement. Biochim. Biophys. Acta 2012, 1818, 574-583.

68. Rowe, E. Lipid chain length and temperature dependence of ethanol-phosphatidylcholine interactions. Biochemistry 1983, 22, 3329-3305.

69. Adachi, T.; Takahashi, H.; Ohki, K.; Hatta, I. Interdigitated structure of phospholipid-alcohol systems studied by X-ray diffraction. Biophys. J. 1995, 68, 1850-1855.

70. Kinoshita, K.; Yamazaki, M. Organic solvents induce interdigitated gel structures in multilamellar vesicles of dipalmitoylphosphatidylcholine. Biochim. Biophys. Acta 1996, 1284, 233-239. 
71. Li, S.J.; Kinoshita, K.; Furuike, S.; Yamazaki, M. Effects of solvents interacting favorably with hydrophilic segments of the membrane surface of phosphatidylcholine on their gel-phase membranes in water. Biophys. Chem. 1999, 81, 191-196.

72. Matsuki, H.; Okuno, H.; Sakano, F.; Kusube, M.; Kaneshina, S. Effect of deuterium oxide on the thermodynamic quantities associated with phase transitions of lipid bilayer membranes. Biochim. Biophys. Acta 2005, 1712, 92-100.

73. Kaneshina, S.; Tamura, K.; Kawakami, H.; Matsuki, H. Effects of pressure and ethanol on the phase behavior of dipalmitoylphosphatidylcholine multilamellar vesicles. Chem. Lett. 1992, 21, 1963-1966.

74. Nemethy, G.; Scheraga, H.A. Structure of water and hydrogen bonding in proteins. I. A model for the thermodynamic properties of liquid water. J. Chem. Phys. 1962, 36, 3382-3400.

75. Nemethy, G.; Scheraga, H.A. Structure of water and hydrogen bonding in proteins. IV. The thermodynamic properties of liquid deuterium oxide. J. Chem. Phys. 1964, 41, 680-689.

76. Ruocco, M.J.; Shipley, G.G. Characterization of the sub-transition of hydrated dipalmitoylphosphatidylcholine bilayers: Kinetics, hydration, and structural study. Biochim. Biophys. Acta 1982, 691, 309-320.

(C) 2013 by the authors; licensee MDPI, Basel, Switzerland. This article is an open access article distributed under the terms and conditions of the Creative Commons Attribution license (http://creativecommons.org/licenses/by/3.0/). 